\title{
Theoretical and Experimental Studies of the Temperature Difference at the Inlet and Outlet of a Pump as a Function of Time and Pressure
}

\author{
M. N. Kostomakhin*, I. M. Makarkin, and N. A. Petrischev \\ Federal Scientific Agro-engineering Center VIM, 1 Institutsky proezd, 5, Moscow, 109428, Russia
}

\begin{abstract}
In the article, the characteristics of the function of temperature difference of a working fluid at the inlet and outlet of gear pumps with different efficiency and at different pressures are theoretically and experimentally investigated. A method is proposed for determining the efficiency of pumps during its operation at variable pressure, based on the hypothesis that the performed useful work of the pump is characterized by the area under the pressure curve, and the lost energy is the area under the temperature difference curve.
\end{abstract}

\section{Introduction}

The thermodynamic method is in essence a statoparametric method, since it requires maintaining a constant steady-state pressure drop and accurate measuring the steady-state temperature difference at the inlet and outlet of the hydraulic unit [1,2], which is not always possible under operating conditions. Of interest is the study of changes in the temperature difference at the pump at variable pressure characteristic of the operating mode.

\section{Materials and methods}

The experiments were carried out on a stand with NSH-32 pumps with different efficiencies.

The ETS 4548-H temperature and HDA 3000 pressure sensors transmitted information to a HYDAC data logger. The polling frequency of the temperature sensors is $1 \mathrm{~Hz}$, the pressure sensor is $2 \mathrm{~Hz}$. Integration and approximation of table data was performed using the Maple-13 program.

The temperature difference at the inlet and outlet of the hydraulic unit as a function of time is characterized not only by the steady-state value of $\Delta \mathrm{T}_{\text {inst }}$, but also by other parameters, such as the growth rate at the initial time and the area under the curve ST at any time t (Figure 1).

Formula (2) characterizes the total lost energy used to heat the working fluid

\footnotetext{
*Corresponding author: tomasss1086@mail.ru
} 


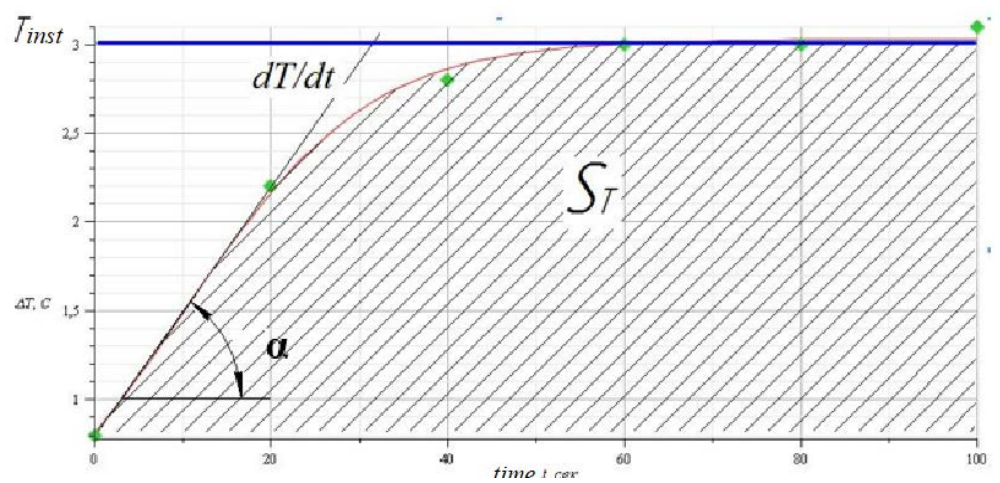

Fig. 1. Function characteristics $\Delta \mathrm{T}(\mathrm{t})$.

These parameters also depend on the efficiency of the hydraulic unit, and knowing them for the reference unit, we can determine the efficiency of the diagnosed one. So, for example, a convenient parameter can be the gradient of the temperature difference at the initial time from the pressure supply

$$
\left.\frac{\partial}{\partial t} \Delta T(t)\right|_{t=0}=\tan (\alpha) \text {. }
$$

To determine it, it is enough to measure the temperature increase for the first 20-30 seconds and calculate the total efficiency using an empirical formula. Figure 11 shows a graph of the dependence of the total efficiency of the gear pump NSH-32-3L on the temperature increase in the first 20 seconds $\left(\left.\frac{\partial}{\partial t} \Delta T(t)\right|_{t=0}=\tan (\alpha)\right)$ and the approximating line at a pressure of $\mathrm{P}=10 \mathrm{MPa}, \mathrm{T} 0=45^{\circ} \mathrm{C}$. The approximating line is constructed based on the results of measuring 5 pumps.

Function approximation $\Delta \mathrm{T}(\mathrm{t})$

The $\Delta \mathrm{T}(\mathrm{t})$ function is well approximated by the logistic curve, which is widely used in chemical kinetics to describe reaction rates depending on the current concentration of reacting components

$$
\frac{\partial}{\partial t} G=k \cdot G\left(1-\frac{G}{K}\right)
$$

where $\mathrm{G}$ - current concentration; $\mathrm{k}$ - characterizes the growth rate, $\mathrm{K}$ - maximum possible concentration (saturated).

By analogy, we can write:

$$
\frac{d}{d t} \Delta T=k \Delta T\left(1-\frac{\Delta T}{\Delta T_{\text {inst }}}\right)
$$

where $\Delta \mathrm{T}$ - current temperature difference; $\mathrm{k}$ - growth rate parameter; $\Delta$ Tdet maximum possible under these conditions (steady-state temperature drop). The solution of this equation is a function

$$
\Delta T=\frac{\Delta T_{\text {inst }} \cdot \Delta T_{0^{\circ}} e^{k t}}{\Delta T_{\text {inst }}+\Delta T_{0} \cdot\left(e^{k t}-1\right)}
$$

After simple transformations, equation (6) takes the form

$$
\begin{aligned}
\Delta T(t) & =\frac{\frac{\Delta T_{\text {inst }}-\Delta T_{0}}{\Delta T_{\text {inst }}-\Delta T_{0}}}{\frac{\Delta T_{0}}{\Delta T_{\text {inst }}-\Delta T_{0}}-e^{-k t}}=\frac{a}{\mathrm{~b}-e^{-k t}} \\
\text { where } \quad \frac{\Delta T_{\text {inst }} \cdot \Delta T_{0}}{\Delta T_{\text {inst }}-\Delta T_{0}} & =a \frac{\Delta T_{0}}{\Delta T_{\text {inst }}-\Delta T_{0}}=b
\end{aligned}
$$




$$
\lim _{t \rightarrow \infty} \frac{\frac{\Delta T_{\text {inst }} \Delta T_{0}}{\Delta T_{\text {inst }}-\Delta T_{0}}}{\Delta T_{0}}=\Delta T_{\text {inst }}-\Delta T_{0}-e^{-k t}
$$

The growth rate parameter $k$ depends on the pressure (Figure 2), the pump efficiency (Figure 3), and the thermophysical parameters of the working fluid. At the same pressure, $\mathrm{k}$ is inversely proportional to the efficiency.

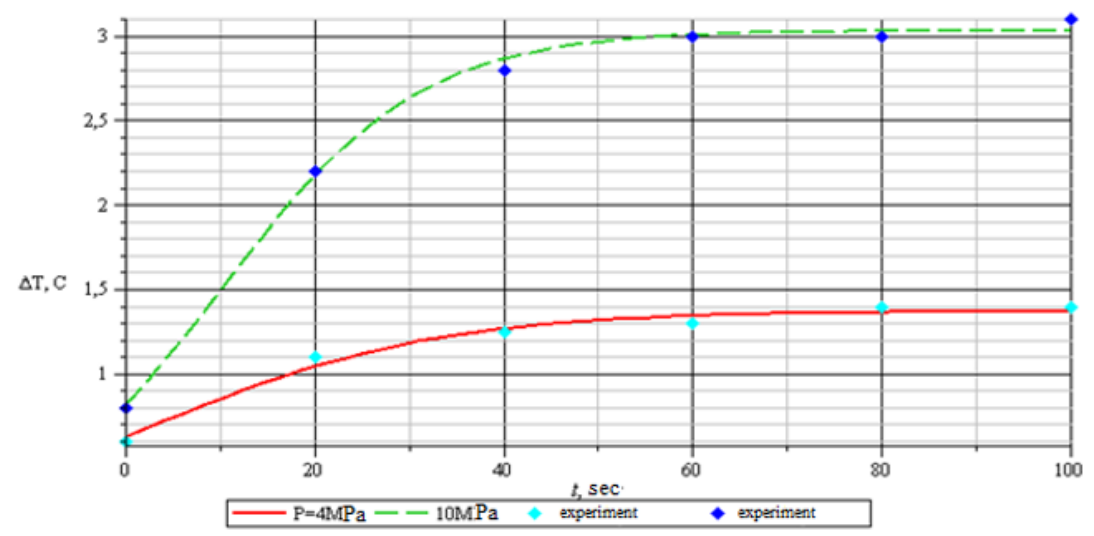

Fig. 2. Graph of changes in the temperature difference at the pump.

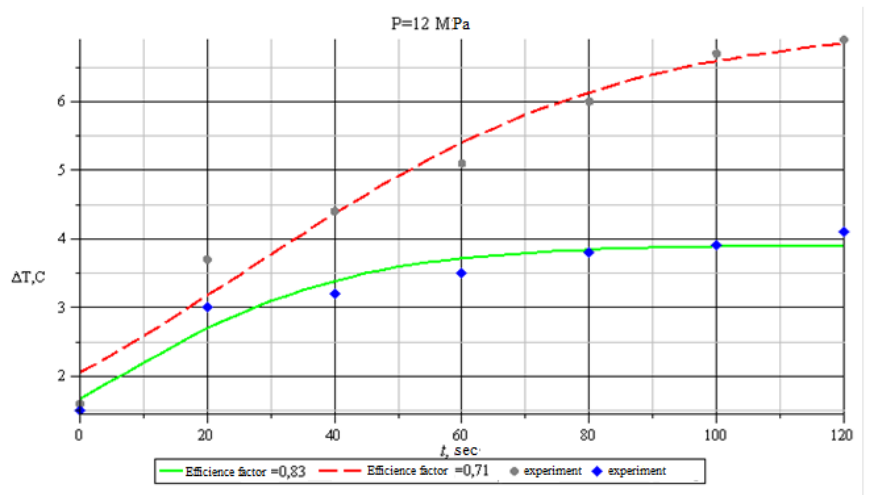

Fig. 3. The change in the temperature difference at the pump from time to time at different efficiencies

The equation of the approximating curve in Figure 3 has the form

For efficiency $=0.71 \frac{2.89}{0.74+\mathrm{e}^{-0.054 \cdot t}}$ and for efficiency $=0,83 \frac{2.86}{0.402+\mathrm{e}^{-0.034 \cdot t}}$

Derivative of the function $\frac{a}{b+\mathrm{e}^{-k \cdot t}}$

Its graph is shown in Figure 4

$$
\frac{\mathrm{d}}{\mathrm{d} t} \frac{a}{b+\mathrm{e}^{-k \cdot t}}=\frac{a k \mathrm{e}^{-k t}}{\left(b+\mathrm{e}^{-k t}\right)^{2}}
$$


At $\mathrm{t}=0$

$$
\frac{\mathrm{d}}{\mathrm{d} t} \frac{a}{b+\mathrm{e}^{-k \cdot t}}=\frac{a \cdot k}{(b+1)^{2}}
$$

This value characterizes the rate of growth of the temperature difference at the initial time and is a diagnostic parameter.

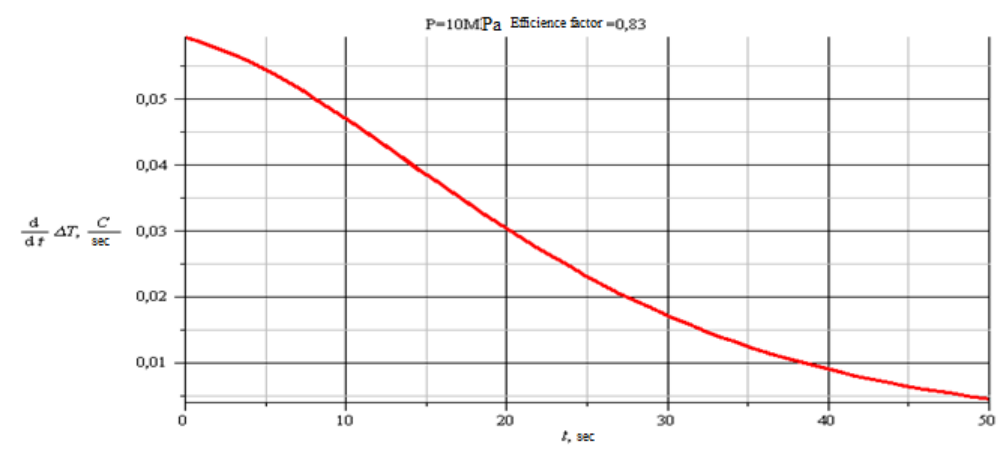

Fig. 4. The rate of change of the temperature difference $\mathrm{d} \Delta \mathrm{T} / \mathrm{dt}$.

A parameter closely related to the pump efficiency is the rate of temperature increase at the output of the dTdischarge/dt, which is well approximated by a straight line, the angle of inclination of which to the time axis is inversely proportional to the pump efficiency, all other things being equal. Figure 5 shows experimental data on three pumps with different efficiency.

The area under the temperature drop curve characterizes the total energy lost

$$
\Delta T(t)=\frac{a}{b-\mathrm{e}^{-k \cdot t}}
$$

At any time the area under the curve is given by the equation

$$
S_{T}(t)=\int_{0}^{t} \Delta T(t) \mathrm{d} t
$$

and all other things being equal, it is a function of the pump efficiency and pressure. The function St $(\mathrm{t})$ changes over time according to the law (Figure 6)

$$
\begin{gathered}
S T(t)=-\frac{a \ln \left(\mathrm{e}^{-k t}\right)}{k b}+\frac{a \ln \left(b+\mathrm{e}^{-k t}\right)}{k b} \\
\lim _{t \rightarrow \infty} S T(t)=\lim _{t \rightarrow \infty}\left(-\frac{a \ln \left(\mathrm{e}^{-k t}\right)}{k b}+\frac{a \ln \left(b+\mathrm{e}^{-k t}\right)}{k b}\right)=a \cdot \frac{\ln (b)}{k \cdot b} ;
\end{gathered}
$$

where $\lim _{t \rightarrow \infty} S T(t)=a \cdot \frac{\ln (b)}{k \cdot b}$ - steady-state growth rate. 
Rate of the temprature growth at the pump outlet

$\mathrm{P}=10 \mathrm{MPa}$

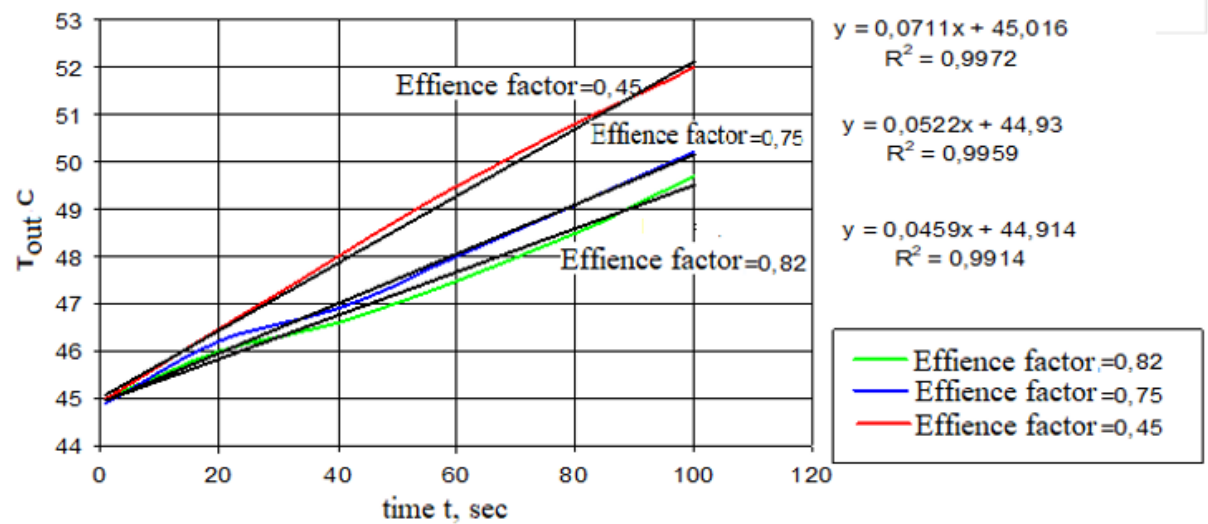

Fig. 5. Temperature change at the outlet of the pump from time to time at different efficiencies.

\section{Results}

Numerous experiments allow us to conclude:

The ratio of the areas under the curve $\Delta \mathrm{T}(\mathrm{t})$ for 2 pumps for any same period of time is a function of the quotient of their total efficiency (Figure 7).

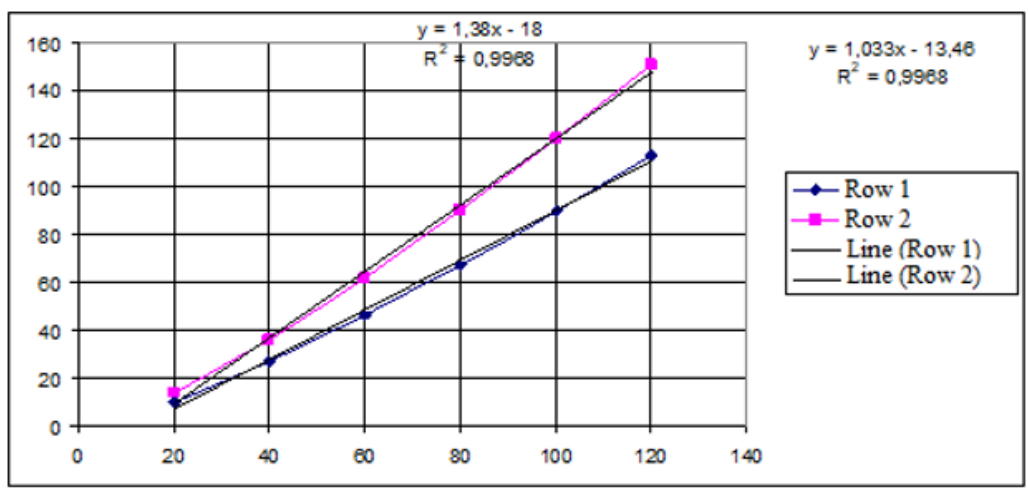

Fig. 6. Graph of the $S T(t)$ function for two pumps. $P=6 \mathrm{MPa}, \eta 1=0.87, \eta 2=0.78$

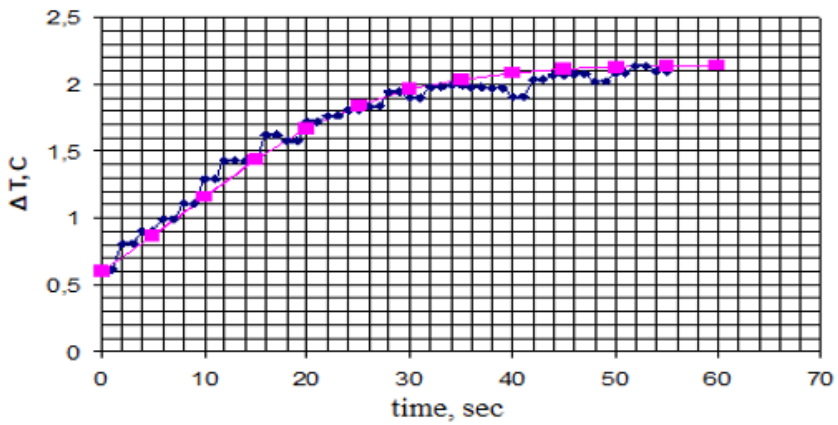

Fig. 7. Experimental and theoretical function of the temperature difference $\mathrm{P}=80 \mathrm{~kg} / \mathrm{cm} 2$, $\mathrm{E}=0,79, \Delta T(t)=\frac{0.832}{0.387+e^{-0,11 \cdot t}}$. 
Regression model of function relationship $\mathrm{P}(\mathrm{t})$ and $\Delta \mathrm{T}(\mathrm{t})$

Source data. Having experimental data on the change in the temperature difference of the working fluid at the pump inlet and outlet, it is possible to obtain an approximating function:

$$
\Delta T(t)=\frac{a}{b-\mathrm{e}^{-k \cdot t}},
$$

use it to calculate the area under the curve - calculate the area under the curve $\Delta T(t)$ in any moment and correlate it with the area under the pressure curve for the same period of time.

We calculate the area ST (t) at different pressures and for different time intervals. We write the result in a table and perform a linear two-factor regression analysis to establish the relationship $\mathrm{ST}=\mathrm{f}(\mathrm{t}, \mathrm{P})$

Example of the regression equation for two pumps: $\mathrm{St} 1(\mathrm{t}, \mathrm{P})=0.9748 * \mathrm{t}+0.7245 * \mathrm{P}-$ 65.335; $\mathrm{ST} 2(\mathrm{t}, \mathrm{P})=1,41 * \mathrm{t}+1,36 * \mathrm{P}-115,9$. Graphical representation of the dependency $\mathrm{St}(\mathrm{t}, \mathrm{P})$ on Figure 8 . The greater the angle of inclination of the plane $\operatorname{St}(\mathrm{t}, \mathrm{P})$ to the plane $0 \mathrm{tP}$, the lower the overall efficiency of the pump.

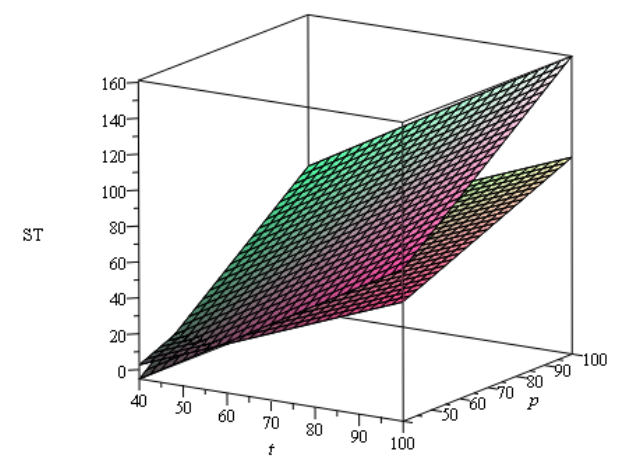

Fig. 8. The area under the temperature curve as a function of two time variables $t$ and pressure $p$. $\eta 1=0,71, \eta 2=0,83$.

Note that in these two equations, the coefficient at $\mathrm{t}$ is greater than the coefficient at $\mathrm{P}$. That is, time is a more significant factor than pressure.

Empirical formula of determining the total efficiency. For the convenience of determining the pump efficiency at variable pressure, it is necessary to construct a twodimensional graph of the dependence of the area under the temperature difference curve $\mathrm{St}=\Sigma \Delta \mathrm{Ti} * \mathrm{ti}$ on the area under the pressure curve at the outlet of the pump $\mathrm{Sp}=\Sigma \mathrm{Pi} \mathrm{ti}^{\mathrm{t}}$.

Let's perform the following method: construct a plane $\mathrm{P}=\mathrm{t}$ (Figure 9). This plane passes through the z-axis and is perpendicular to the ORt plane. We also construct several surfaces $\mathrm{P} * \mathrm{t}=$ const (Figure 9 shows the surfaces for the cases $\mathrm{P} * \mathrm{t}=400 \mathrm{MPa}^{*} \mathrm{~s}$ and $\mathrm{P} * \mathrm{t}=800 \mathrm{MPa}^{*} \mathrm{~s}$ ). These surfaces are hyperbolic cylinders. Now we take the plane $\mathrm{P}=\mathrm{t}$ as a new coordinate plane, along the abscissa axis of which we will plot the areas under the pressure curve Sp, and along the ordinate axis - the area under the temperature difference curve St (Figure 10) The plane St $(t, P)$, obtained as a result of regression analysis of experimental data, intersects the plane $\mathrm{P}=\mathrm{t}$ in a straight line, the angle of inclination of which to the abscissa axis is inversely proportional to the total efficiency of the pump. 


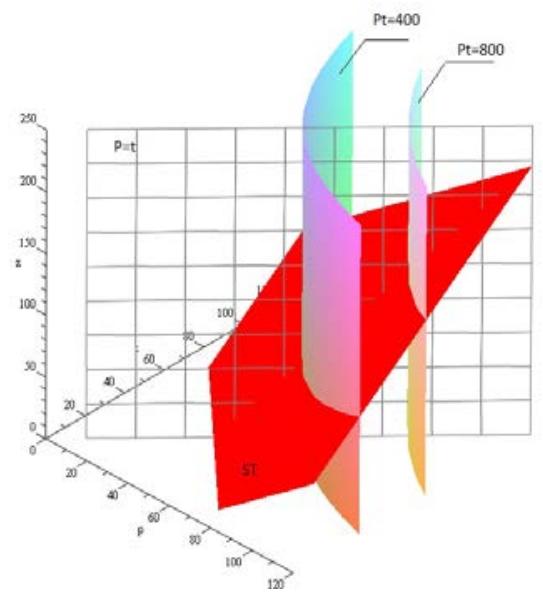

Fig. 9. Surfaces $\mathrm{St}(\mathrm{P}, \mathrm{t}), \mathrm{P} * \mathrm{t}=$ const and $\mathrm{P}=\mathrm{t}$.

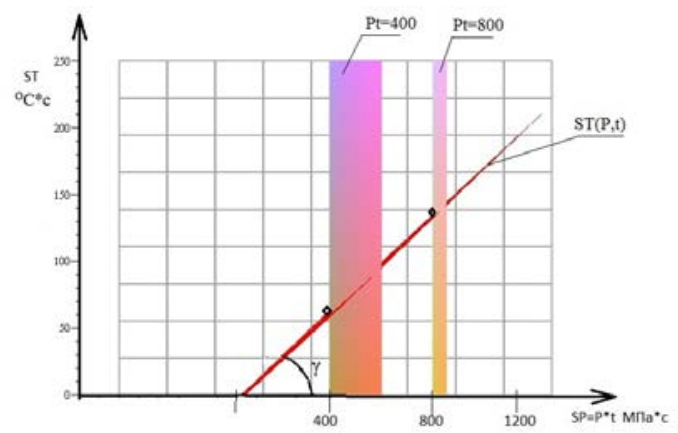

Fig. 10. To determine the efficiency of the pump.

The results of processing the experimental data for three different pumps are shown in Figure 11.

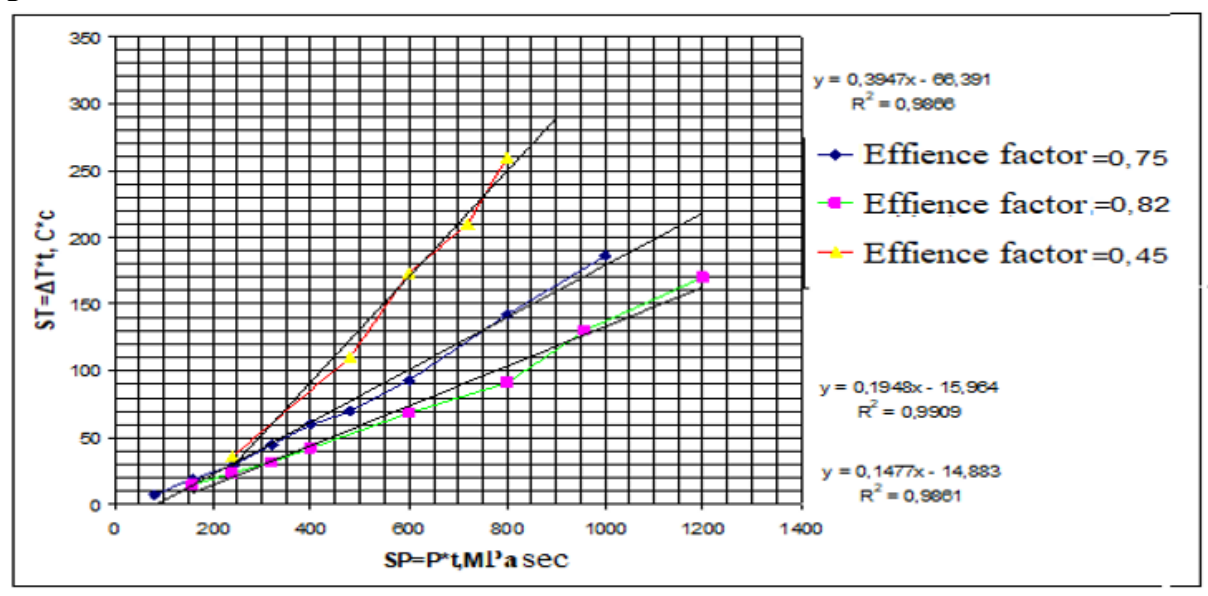

Fig. 11. Graphs of the dependence of $\mathrm{ST}=\mathrm{f}(\mathrm{SP})$ for three different pumps.

The approximation of the dependence of the total efficiency on the angle of inclination $\gamma$ (Figure 10,11) gives the dependence

$$
\eta=-1,45 \cdot \operatorname{tg} \gamma+1
$$


Taking $\operatorname{tg} \gamma=\frac{S T}{S P}$, we obtain an empirical formula for determining the total efficiency:

$$
\eta=-1,45 \cdot \frac{S T}{S P}+1
$$

or

$$
\eta=-1,45 \cdot \frac{\int_{0}^{t} \Delta T(t) \cdot d t}{\int_{0}^{t} P(t) \cdot d t}+1
$$

The integral in the numerator of formula (18) characterizes the lost energy spent on heating the working fluid, and the integral in the denominator characterizes the useful work performed by the pump.

\section{Conclusions}

The possibility of determining the total efficiency of hydraulic pumps by the thermodynamic method under an arbitrary loading mode characteristic of the functioning of hydraulic systems is shown. This will allow us to create a simple system for early detection of faults mounted on the hydraulic system.

\section{References}

1. Sholom, Anatoly Mikhaylovich. Development and research of a thermodynamic method of diagnosing volumetric hydraulic machines in order to manage their quality: Moscow, 1980.

2. Grinchar N.G. RGOTUPS. In collection of works «Modern problems of improving the work of railway transport», p. 152-156 (1999). 